\title{
Erratum to: ALG6-CDG: a recognizable phenotype with epilepsy, proximal muscle weakness, ataxia and behavioral and limb anomalies
}

\author{
Eva Morava ${ }^{1,2} \cdot$ Vera Tiemes $^{3,4} \cdot$ Christian Thiel $^{5} \cdot$ Nathalie Seta $^{6} \cdot$ Pascale de Lonlay $^{7} \cdot$ Hans de Klerk $^{8}$. \\ Margot Mulder $^{9}$ - Estela Rubio-Gozalbo ${ }^{10}$ • Gepke Visser ${ }^{11}$ • Peter van Hasselt ${ }^{11}$ • Dafne D. G. Horovitz ${ }^{12}$ • \\ Carolina Fischinger Moura de Souza ${ }^{12}$ - Ida V. D. Schwartz ${ }^{12}$. Andrew Green ${ }^{13}$ • Mohammed Al-Owain ${ }^{14}$. \\ Graciella Uziel $^{15}$ - Sabine Sigaudy ${ }^{16}$ - Brigitte Chabrol ${ }^{17}$. Franc-Jan van Spronsen ${ }^{18}$ - Martin Steinert ${ }^{19}$. \\ Eleni Komini ${ }^{20}$ • Donald Wurm ${ }^{21}$ - Andrea Bevot ${ }^{22}$ - Addelkarim Ayadi $^{6}$ - Karin Huijben ${ }^{4}$ - Marli Dercksen ${ }^{23}$. \\ Peter Witters $^{1}$ • Jaak Jaeken ${ }^{1}$ • Gert Matthijs ${ }^{24}$ • Dirk J. Lefeber ${ }^{4,25} \cdot$ Ron A. Wevers ${ }^{4}$
}

(C) SSIEM 2016

\section{Erratum to: J Inherit Metab Dis DOI 10.1007/s10545-016-9945-x}

The name of the author Christian Thiel was rendered wrongly in the original publication but has since been corrected.

The online version of the original article can be found at http://dx.doi. org/10.1007/s10545-016-9945-x.

\section{Eva Morava \\ eva.morava@uzleuven.be \\ $\triangle$ Ron A. Wevers}

ron.wevers@radboudumc.nl

1 Center for Metabolic Diseases, Department of Pediatrics, University Hospitals Leuven, Leuven, Belgium

2 Tulane University Medical School, Hayward Genetics Center, New Orleans, LA, USA

3 Department of Pediatrics, Radboud University Medical Center, Nijmegen, The Netherlands

4 Translational Metabolic Laboratory, Department Laboratory Medicine, Radboud University Medical Center,

Nijmegen, The Netherlands

5 Center for Child and Adolescent Medicine, Kinderheilkunde I, University of Heidelberg, Heidelberg, Germany

6 Biochimie Métabolique Hôpital Bichat-Claude Bernard, Paris, France

7 Reference Center of Metabolism, Necker-Enfants Malades Hospital, APHP, Imagine Institute, University Paris-Descartes, Paris, France

8 Department of Pediatrics, Erasmus MC - University Medical Center Rotterdam, Emma Hospital, Rotterdam, The Netherlands

9 Department of Pediatrics, Free University Amsterdam, Amsterdam, The Netherlands

10 Department of Pediatrics and Laboratory Genetic Metabolic Diseases, University of Maastricht, Maastricht, The Netherlands

11 Wilhelmina Children's Hospital, University Medical Center Utrecht, Utrecht, The Netherlands
12 Medical Genetics Service, Porto Alegre, RS, Brazil

13 National Centre for Medical Genetics, Dublin, Ireland

14 Department of Medical Genetics, King Faisal Specialist Hospital and Research Centre, Riyadh, Saudi Arabia

15 University of Milan, Institute of Neurology, Milan, Italy

16 Département de Génétique Médicale, Hôpital Timone Enfant, Marseille, France

17 Neuropediatrics Unit, Childrens Hospital CHU Timone, Marseille, France

18 Division of Metabolic Diseases, Beatrix Children's Hospital, University Medical Center of Groningen, University of Groningen, Groningen, The Netherlands

19 Sozialpädiatrisches Zentrum, Neuropädiatrie, Klinik für Kinder- und Jugendmedizin, Dortmund, Germany

20 Kinderklinik Villingen, Schwarzwald-Baar-Klinikum, Villingen, Germany

21 Department of Pediatrics, Klinikum Saarbrücken, Saarbrücken, Germany

22 Department of Pediatric Neurology and Developmental Medicine, Universal Children's Hospital Tübingen, Tübingen, Germany

23 Centre for Human Metabolomics, North-West University, Potchefstroom, South Africa

24 Laboratory for Molecular Diagnosis, Center for Human Genetics, University of Leuven, Leuven, Belgium

25 Department of Neurology, Radboud University Medical Center, Nijmegen, The Netherlands 